\title{
ROMANIAN
}

NEUROSURGERY

Vol. XXXIV | No. 4 December 2020

\section{Intradural migration of bullet in vertebra corpus after meningitis}

Halil Ibrahim Gündüz,

Turan Kandemir 


\title{
Intradural migration of bullet in vertebra corpus after meningitis
}

\author{
Halil İbrahim Gündüz ${ }^{1}$, Turan Kandemir ${ }^{2}$ \\ 1 Eskişehir City Hospital, Department of Neurosurgery, \\ Eskisehir, TURKEY \\ 2 Yunus Emre State Hospital, Department of Neurosurgery, \\ Eskisehir, TURKEY
}

\begin{abstract}
In a gunshot injury, the spinal cord of the thoracic region is usually the most affected and damaged part of the body. In most cases, the bullet cannot be removed without causing more damage to the injury. Over time, the bullet tends to travel in different areas of the body. Moreover, cases on bullet movements in the spinal canal were reported in the literature. In this study, we reviewed the diagnosis and treatment of a 27-year-old male patient with a bullet detected in his vertebra corpus, which is caused by a gunshot injury. During the follow-up period, an intradural migration of the bullet from the vertebra corpus was observed. Furthermore, we performed surgery to prevent any future neural damage. In this study, we focused on a case with a gunshot injury, presenting an intradural migration of a bullet from the vertebra corpus after meningitis.
\end{abstract}

\section{INTRODUCTION}

Gunshot injuries (GSI) are the third most common cause of spinal cord injuries after traffic accidents and falls from height. Although the incidence of spinal cord injuries caused by GSI changes according to the countries' level of development, the most common causes of it are suicides, accidents and attacks. Moreover, in developed countries, the approximate percentage of spinal cord injuries caused by GSI among all kinds of spinal cord injuries was reported to be $15 \%^{[1,2]}$.

Aside from the aforementioned damages on the spinal cord, secondary injuries, such as the degradation of the spinal cord vascularisation, autoregulation deficiency and hypotension, also worsen the neurological presentation due to systemic effects ${ }^{[3]}$.

In the literature, cases of bullets travelling through the intraspinal canal were reported. In our case, the bullet was first stuck in the vertebra corpus, and removing it through manipulation was not feasible during the surgical operation. But in the follow-up period, it was observed that the bullet had left the corpus and travelled through the spinal canal after meningitis.

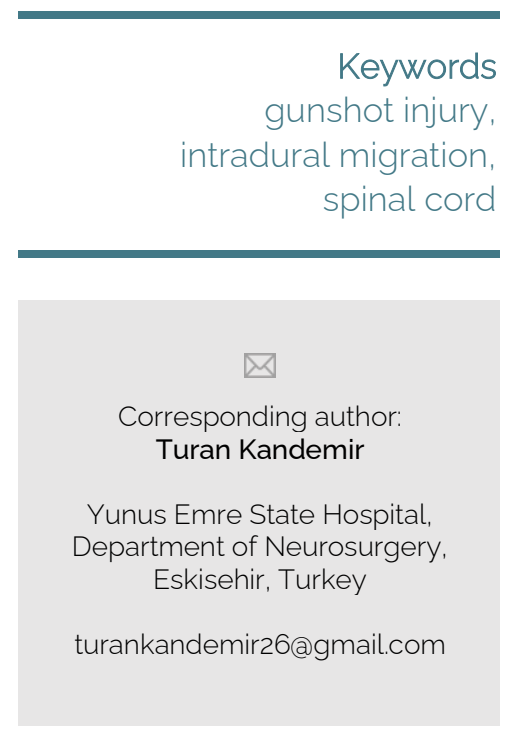

Copyright and usage. This is an Open Access article, distributed under the terms of the Creative Commons Attribution Non-Commercial No Derivatives License (https://creativecommons org/licenses/by-nc-nd/4.0/) which permits noncommercial re-use, distribution, and reproduction in any medium, provided the original work is unaltered and is properly cited

The written permission of the Romanian Society of Neurosurgery must be obtained for commercial re-use or in order to create a derivative work.

ISSN online 2344-4959 (C) Romanian Society of Neurosurgery

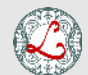

First published December 2020 by London Academic Publishing www.lapub.co.uk 


\section{CASE PRESENTATION}

A 27-year-old male patient was admitted to the emergency service following the gunshot injury in the chest area. A chest tube was then inserted as the patient had hemopneumothorax. A hole in the patient's body due to the bullet which entered his thorax from right axillary lower region was observed. Based on the neurological examination, the patient had paraplegia in the lower extremity, and anaesthesia was administered below T10; moreover, an anal sphincter reflex was not observed. The steroid protocol treatment from the NASCIS protocol was performed. A bullet was stuck in the T10 corpus was observed using the spinal tomography (Figure 1). Therefore, an urgent surgery was performed. During the surgery, it was observed that the dura and medulla were partially disintegrated. Two-third of the bullet was in the T10 corpus. However, the bullet could not be removed despite further manipulation. The operation was then ended following duraplasty. As no change in the postoperative neurological examination was observed, the patient was required to undergo physical therapy and rehabilitation and was discharged afterwards.

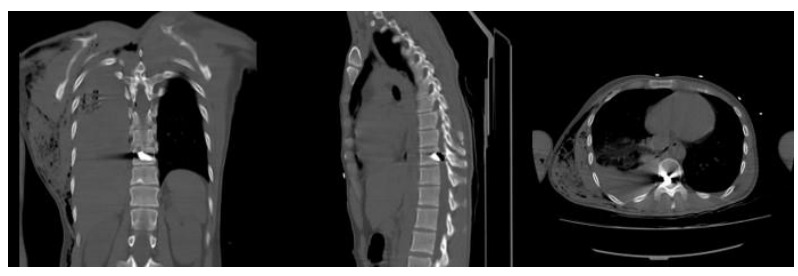

Figure 1. Image of the bullet in the T10 corpus following the gunshot injury.

Due to wound drainage and high fever, the patient was re-admitted a month after the discharge. A meningeal irritation was observed, and the white blood count and C-reactive protein were high. Due to the presence of meningitis, antibiotherapy was started. Moreover, the patient was reoperated since serous wound discharge continued to persist. During the surgery, it was observed that the discharge was coming from an intradural distance. The bullet was stuck in the corpus could not be removed through manipulation. Duraplasty was then performed again, and the wound discharge ended postoperatively. After the antibiotherapy was completed and the wound recovered completely, the patient was discharged and was required to undergo physical therapy and rehabilitation.
Based on the medical imaging performed nearly a year later, it was observed that the bullet in the T10 corpus was dislocated and moved in an intradural distance; it reached the S1 level (Figure 2). Moreover, the neurological deficit of the patient regressed, and the muscle strength of the lower right extremity was evaluated as $4 / 5$ and the lower left extremity $2 / 5$. The patient underwent surgery in order to remove the bullet. During the operation, it was observed that the bullet had reached the $L 4$ level when checked based on the results of the fluoroscopy (Figure 3). L4 total laminectomy was performed after opening the L4 level. When the dura was opened, it was observed that the bullet was in the L3 level. When the patient was in a reverse Trendelenburg position, the bullet moved towards the dural opening and was removed. The dura was closed via primary suturing. Furthermore, no additional deficit was observed in the postoperative neurological examination.

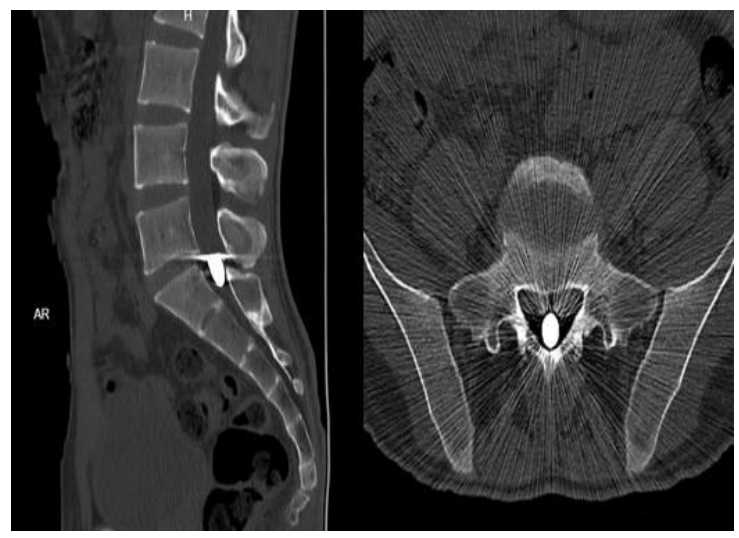

Figure 2. CT image showing the bullet at the S1 level.

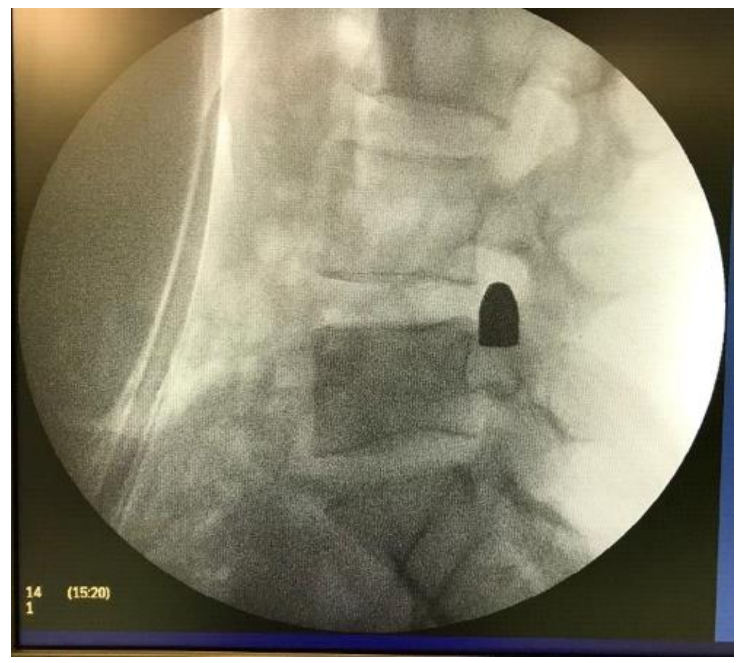

Figure 3. Fluoroscopy image before intraoperative laminectomy 


\section{DISCUSSION}

Spinal gunshot injuries occur mostly in the thoracic region. Another common place for it to occur is the lumbar region, with the cervical region being the less common ${ }^{[4]}$. Injuries in the cervical region have a more mortal course. In the thoracic region, the spinal canal/cord ratio is lower and the neural damage is higher compared with that in other levels.

In the literature, studies showing a bullet migration in the intervertebral disc level and in the paraspinal mass tissue into the spinal canal were included. Kuijlen et al. presented a case of bullet migration from the paraspinal muscles to the spinal canal at the L3 level ${ }^{[5]}$. Conway et al. presented a case showing a cauda equina development after a bullet migration from the $L 4$ to $L 5$ intervertebral disc level ${ }^{[6]}$. Ceylan et al. presented a case who had back pain caused by a bullet migration anteriorly between the L2 and L3 intervertebral disc levels ${ }^{[7]}$. In our case, the bullet was stuck in the T10 corpus and migrated through the spinal canal in a span of nearly 1 year since the injury. In our case, in contrast to the other cases, two-third of the bullet was stuck in the vertebra corpus which could not be removed by manipulation during the two surgical operations performed, and the bullet spontaneously migrated to the canal following the patient's meningitis treatment.

In a gunshot injury, leaving the bullet inside the body rarely causes infection, neurological deficit and lead poisoning ${ }^{[8]}$. In the case of our patient, the infection occurred followed by a to cerebrospinal fluid (CSF) leakage after the first operation. Moreover, gunshot injury treatment is still a disputed and complex subject. Surgery is necessary if CSF leakage, progressive neurological deficit and infection formation co-occur with the infection. Aside from gravity, respiratory movements and CSF fluidity are also factors that affect the bullet movement inside the spinal canal. A neurological deficit may also occur if the bullets move in the spinal canal; in our case, no signs of newly developed neurological deficits were observed. However, a neurological deficit may develop later due to the fibrotic reactions forming in the pia and arachnoid. It was also shown that axon and myelin damage and lead and copper implantation causes induces gliosis in the spinal cord ${ }^{[9,10]}$. Therefore, we decided to remove the bullet piece although our patient lacked any symptom or additional neurological finding.
Computed and direct tomography are usually the first diagnostic options for gunshot injuries. Kafadar et al. reported that a magnetic resonance imaging (MRI) can be performed since the bullet is covered with non-ferromagnetic metals just like copper in low-speed gunshot injuries ${ }^{[11]}$. Although MRI is the appropriate option for the evaluation of neurological tissue damage and causes less artefact compared to computed tomography (CT), its use is limited depending on the patient. Since such cases are required to undergo an urgent operation, a ballistic examination cannot be performed for the bullet as MRI is more commonly used. Moreover, the patient did not have an MRI scan since we did not have the sufficient information about the bullet structure. When our patient was positioned on the operation table, the results of the endoscopy showed that the bullet was moving. The bullet which was at the S1 level based on the preoperative CT scan had reached the L4 level, and we determined our incision accordingly. In the case presented by Genç et al., the bullet moved intraoperatively, and its location was determined via ultrasonography ${ }^{[12]}$. We then performed laminectomy after determining the location of the bullet via endoscopy. However, we did not have to perform a long-level laminectomy and refrained from stabilisation.

Based on the findings of this study, it should be noted that despite being stuck in the bone, most foreign objects can move in later stages. Overall, no neurological deficit following the bullet migration was observed in our patient and necessary measures were taken to prevent any possible neurological deficit. In addition, the individual who caused the injury was also identified by the researchers.

\section{References}

1. Aarabi B, Alibaii E, Taghipur M, Kamgarpur A. Comparative Study of Functional Recovery for Surgically Explored and Conservatively Managed Spinal Cord Missile Injuries. Neurosurgery 1996;39(6):1133-40.

2. Young J. Spinal cord injury statistics: experience of the regional spinal cord injury systems. 1982.

3. Bono CM, Heary RF. Gunshot wounds to the spine. The Spine Journal 2004;4(2):230-40.

4. Calik S, Calik M, Esme H. Intraspinal Bullet Migration: A Rare Case Report. CHEST 2017;152(4):A44.

5. Kuijlen JM, Herpers MJ, Beuls EA. Neurogenic Claudication, a Delayed Complication of a Retained Bullet. Spine 1997;22(8):910-914. 
6. Conway JE, Crofford TW, Terry AF, Protzman RR. Cauda equina syndrome occurring nine years after a gunshot injury to the spine. A case report. JBJS 1993;75(5):760763.

7. Ceylan D, Cosar M. Migration of a Bullet in the Lumbar Intervertebral Disc Space Causing Back Pain. Neurologia medico-chirurgica 2008;48(4):188-90.

8. Yoshida GM, Garland D, Waters RL. Gunshot wounds to the spine. Orthop Clin North Am 1995;26(1):109-16.

9. Bordon G, Burguet Girona S. Gunshot wound in lumbar spine with intradural location of a bullet. Case Rep Orthop 2014;2014:698585.
10. Esnal-Baza E, Zaldua-Unanue M, Etxebarria-Foronda I. Neurological Symptoms Secondary to the Intraspinal Migration of a Bullet. Revista Española de Cirugía Ortopédica y Traumatología (English Edition) 2007;51(6):351-3.

11. Kafadar AM, Kemerdere R, Isler C, Hanci M. Intradural migration of a bullet following spinal gunshot injury. Spinal Cord 2006;44(5):326-9.

12. Genç A, Usseli MI, Pamir MN. When the bullet moves! Surgical caveats from a migrant intraspinal bullet. Neurologia i Neurochirurgia Polska 2016;50(5):387-91. 